\title{
The electron-hole superfluidity in two coaxial nanotubes
}

\author{
Oleg L. Berman, Ilya Grigorenko, Roman Ya. Kezerashvili \\ Physics Department, New York City College of Technology, \\ The City University of New York, Brooklyn, NY 11201, USA
}

(Dated: May 11, 2018)

\begin{abstract}
The superfluid phase and Coulomb drag effect caused by the pairing in the system of spatially separated electrons and holes in two coaxial cylindrical nanotubes are predicted. It is found that the drag resistance as a function of temperature experiences a jump at the critical temperature and can be used for the manifestation of the superfluid transition. It is demonstrated that at sufficiently low temperatures the order parameter and free energy density exhibit a kink due to the electron-hole asymmetry that is controlled by the radii of the nanotubes.
\end{abstract}

\section{INTRODUCTION}

The superfluid state for a dense system of spatially separated electrons and holes in two parallel layers was predicted in Ref. 1, and physical properties of this state have been studied ${ }^{2-4}$. The peculiarity of such systems is that the pairing between the carriers occurs not via the usual weak electron-phonon interaction mechanism like in conventional superconductors, but through the much stronger electron-hole Coulomb attraction. When the strength of the mutual attraction in dense systems is sufficiently weak, a standard Bardeen-Cooper- Schrieffer (BCS) description ${ }^{5}$ is applicable. Another limiting case is characterized by a sufficiently strong interaction in dilute systems, when fermions form bounded pairs, which can be described as composite bosons (indirect excitons), which undergo Bose-Einstein condensation (BEC). In the both limits the Coulomb attraction between electrons and holes can introduce Coulomb drag that is a process in spatially separated conductors, which enables a current flowing in one of the conductors to induce a voltage drop in the other one. In the case when the second conductor is a part of closed circuit, the induced current flows. The experimental observation of the exciton condensation and perfect Coulomb drag was claimed recently for the spatially separated electrons and holes in GaAs/AlGaAs coupled quantum wells at the presence of high magnetic field perpendicular to the quantum wells 6 . A steady transport current of electrons driven through one quantum well was accompanied by an equal current of holes in the other. The intermediate regime, the so called BCS-BEC crossover, is an interesting phenomenon by itself7 7 , but it is beyond the present study. The BCS regime is achieved for relatively high fermionic densities 1 and it is a subject of the present work.

In Ref. 2 the authors discussed the drag of holes by electrons in a semiconductor-insulator-semiconductor structure. The prediction was that for two conducting layers separated by an insulator there will be a drag of carriers in one layer due to the direct Coulomb attraction with the carriers in the other layer. The Coulomb drag effect in the electron-hole two-layer BCS system was also analyzed in Ref. 3. If the external potential difference is applied to one of the layers, it will cause the electric cur- rent in the other layer. The current in another layer will be initiated due to the correlations between electrons and holes at temperatures below the critical one. Let's mention also that the theory of the drag effects in the system of spatially separated electrons and excitons in an optical microcavity developed in Refs. 9,10 predicts that at low temperature an electron current induces the polariton flow, while the electron current dragged by the polariton flow is strongly suppressed below polariton superfluid transition temperature. This demonstrates the asymmetry of the drag processes in the system. In all above mentioned studies possible asymmetry between electrons and holes excitation spectra is caused by the difference between the effective masses of the carriers. In this work we present the study of the asymmetry in the excitation spectra of electrons and holes and its effect on the Coulomb the drag process due to different radii of two coaxial nanotubes.

Let us consider a system of two coaxial cylindrical nanotubes separated by a dielectric with the spatially separated electrons and holes confined on each nanotube, as shown in Fig. 1. We study the formation of the superfluid phase resulting in the electron-hole Coulomb drag effect in this system. The electrons on the outer nanotube and holes on the inner one have different excitation spectra due to the different radii of the nanotubes. As a result, the conductivities for the inner and outer nanotubes can substantially differ from each other and also strongly depend on the radii of the nanotubes. By measuring the drag conductivity as a function of the temperature one can observe the superfluid transition in the system, and by measuring the jump in the drag coefficients one can obtain the critical temperature of the BCS phase transition causing the superfluidity. The paper is organized in the following way. In Sec. II we discuss the particularities of the physical properties of the system originated from the cylindrical geometry of two coaxial nanotubes. The BCS state for the spatially separated electrons and holes in two coaxial nanotubes is described in Sec. III, In Sec. IV the calculation of the transconductivity coefficients is presented. Finally, the discussion of the results and conclusions follow in Sec. V. 


\section{TWO COAXIAL NANOTUBES}

We consider a system of two coaxial cylindrical nanotubes and assume that the inner nanotube is doped by holes, while carriers on the outer nanotube are electrons. The geometry of two coaxial nanotubes leads to significant differences compared to a system of two parallel plane layers ${ }^{1,3,4}$, and promises much richer physics, compared to the plane geometry. Unlike the case of two plane layers, the conductivities for the inner and outer nonotubes can differ because of their different curvatures. The quantum confinement in the coaxial nanotubes system may result in enhancement of the order parameter for smaller radii of the nanotubes. Similar confinement effects were studied in thin superconducting layers $\frac{11}{12}$ and in metallic cylindrical superconducting nanowires 12 .

For two coaxial cylindrical nanotubes there are two interesting cases: i) both nanotubes have equal amount of electrons and holes; ii) both nanotubes have equal densities of electrons and holes. If the numbers of electrons and holes are equal, as shown in Fig. 17, the carrier concentrations and the chemical potentials for the electrons and holes on the outer and the inner nanotubes will be different. One would expect to observe a lower chemical potential in the outer nanotube. Even though at low temperatures all electrons and holes can be paired, it is expected that the mismatch between the chemical potentials $\mu_{e} \neq \mu_{h}$ may significantly reduce the critical temperature of the superconducting transition. For sufficiently large mismatch between the chemical potentials, comparable to the order parameter, the BCS transition may be impossible. One has also to note that even in the case of perfectly equal carrier concentrations $\rho_{e}=\rho_{h}$ the chemical potentials may be slightly different due to the effect of the different curvatures of the nanotubes. This difference is expected to be noticeable for relatively small, up to several nanometers, but different radii of the nanotubes.

In the case of equal densities of the carriers on each nanotube, the total number of the carriers on the outer nanotube will be higher then on the inner one, as this is shown in Fig. 10. At low temperatures this will result in a considerable amount of the carriers on the outer nanotube (electrons), which can not find their pairs on the inner nanotube (holes). In this case the conductivity in the system will be defined by the paired and unpaired components of the system. One has to note that we consider only the case of relatively weak imbalance between the carriers. A larger difference between the numbers of the electrons and holes may lead to a phase separation between paired and unpaired components 13 .

Below we focus on the case of equal densities of the carriers on each nanotube when the system comprises of three components: the ground state of electron-hole pairs (the superfluid component), the quasiparticle excitations above the ground state (the normal component) and the unpaired normal electron component on the outer nanotube. The contribution to the conductiv-

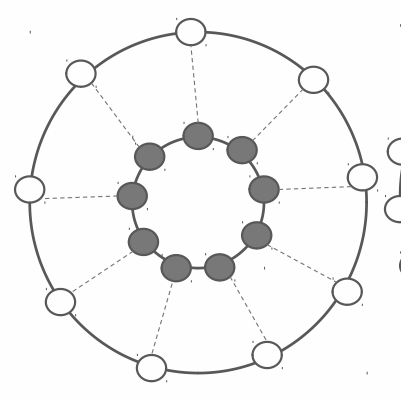

a)

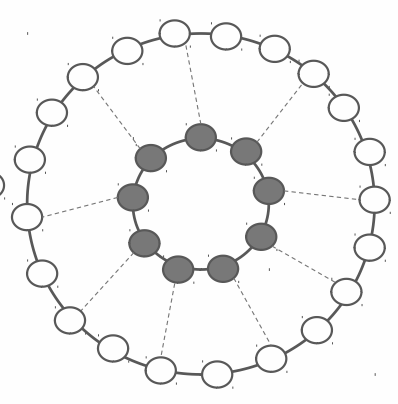

b)
FIG. 1: The distribution of holes (dark circles) on the inner nanotube and electrons (white circles) on the outer one. a) the case of equal amount of electrons and holes, resulting in different carrier densities; b) the case of equal densities of holes and electrons. Note that not all electrons on the outer nanotube can find a partner hole.

ity due to the unpaired component on the outer nanotube may be significant even at zero temperature. Let us consider a finite amount of unpaired electrons on the outer nanotube, with the conductivity $\sigma_{e e}^{u n}$. We assume that in the first approximation the unpaired electron component moves with the same average velocity as the normal component ${ }^{14}$. This will result in a greater current on the outer nanotube, than on the inner one. The difference will correspond to the flow of the unpaired component. In this case the effective electron conductivity coefficient (the conductivity of the outer nanotube) can be estimated as $\sigma_{e e}^{e f f}=\sigma_{e e}+\sigma_{e e}^{u n}$. The conductivity for the unpaired component can be calculated as $\sigma_{e e}^{u n}=\frac{e^{2} \rho_{u n} \tau}{m_{e}}$, where $m_{e}$ is the mass of an electron, and $\tau$ is the characteristic scattering time. The density of the unpaired electrons is simply $\rho_{u n}=\rho \frac{R_{\max }-R_{\min }}{R_{\max }}$. In the last expression $\rho$ is the concentration of the carriers on both nanotubes and $R_{\max }, R_{\min }$ are the radii of the outer and inner nanotube, respectively.

Note that if the carriers density is not very high, the unpaired electrons may strongly interact with Cooper pairs. In two coaxial nanotubes in the dilute limit the electrons and holes form electron-hole bounded statesindirect excitons $\stackrel{15}{\underline{1}}$. Moreover in the dilute limit if the numbers of electrons and holes are different, in the quantum wells the carriers can even form bounded states of trions $16-19$. In dilute limit the trions can be formed in the system of two coaxial nanotubes as well. In this case if the outer nanotube is doped by electrons we have to deal with negative trions $X^{-}$, while if it is doped by holes the positively charged trions $X^{+}$are formed.

In the case of unequal electron and hole densities resulting in unbalanced chemical potentials, the system behavior becomes more complicated. The BCS superfluid phase is energetically unfavored, if the difference in the chemical potentials exceeds $\Delta_{0} / \sqrt{2}$, where $\Delta_{0}$ is the BCS 
gap function at $T=0 \mathrm{~K}$ and $\mu_{e}=\mu_{h}$. The system is expected to form inhomogeneous superfluid phases, where the Cooper pairs have non-zero total momentum. This is the case of the Larkin-Ovchinnikov-Fulde-Ferrel phase, discussed, for example, in Refs $\underline{20}-\underline{22}$. In the present study we assume equal chemical potentials for the electrons and holes that also leads to close electron and hole densities.

\section{THE ELECTRON-HOLE BCS STATE IN TWO COAXIAL NANOTUBES}

Let us consider a system of two thin cylindrical nanotubes of radii $R_{\min }$ and $R_{\max }$, and assume that the nanotubes are doped in such way that the electrons are the carriers on the outer nanotube, and holes are the carriers on the inner one. The effective Hamiltonian of this system can be written as 1

$$
\begin{array}{r}
H_{e f f}=\sum_{\mathbf{p}}\left(\xi_{\mathbf{p}}-\mu_{e}\right) b_{\mathbf{p}}^{\dagger} b_{\mathbf{p}}+\sum_{\mathbf{p}^{\prime}}\left(\xi_{\mathbf{p}^{\prime}}^{\prime}-\mu_{h}\right) a_{\mathbf{p}^{\prime}}^{\dagger} a_{\mathbf{p}^{\prime}}+ \\
+\sum_{\mathbf{p}, \mathbf{p}^{\prime}}\left[\Delta_{\mathbf{p}, \mathbf{p}^{\prime}} b_{\mathbf{p}}^{\dagger} a_{-\mathbf{p}^{\prime}}^{\dagger}+H . c .\right],
\end{array}
$$

where $a_{\mathbf{p}^{\prime}}$ is the operator of annihilation of a hole on the inner nanotube, and $b_{\mathbf{p}}$ is the operator of annihilation of an electron on the outer nanotube. The single-particle eigenenergies of the electrons on the outer nanotube are given by

$$
\xi_{\mathbf{p}}=\frac{|\mathbf{p}|^{2}}{2 m_{e}}=\frac{\hbar^{2}}{2 m_{e}}\left[\frac{N^{2}}{d^{2}}+\frac{m^{2}}{R_{\max }^{2}}+k_{z}^{2}\right],
$$

that correspond to the linear motion of an electron with the momentum $p_{z}=\hbar k_{z}$ and the angular motion with the angular momentum $L_{e}=R_{\max } p_{\phi}=R_{\max } \times \hbar \frac{m}{R_{\max }}=$ $\hbar m, m=0, \pm 1, \pm 2, \ldots$. Similarly the single-particle eigenenergies of the holes confined on the inner nanotube are presented as

$$
\xi_{\mathbf{p}^{\prime}}^{\prime}=\frac{\left|\mathbf{p}^{\prime}\right|^{2}}{2 m_{h}}=\frac{\hbar^{2}}{2 m_{h}}\left[\frac{N^{2}}{d^{2}}+\frac{m^{2}}{R_{\min }^{2}}+k_{z}^{\prime 2}\right] .
$$

The angular momentum of a hole is $L_{h}=R_{\min } p_{\phi}^{\prime}=$ $R_{\text {min }} \times \hbar \frac{m}{R_{\min }}=\hbar m, m=0, \pm 1, \pm 2, \ldots$. In Eqs.(2) and (3) $m_{e}$ and $m_{h}$ are the effective masses of electrons and holes and the expression $\frac{\hbar^{2}}{2 m_{e, h}} \frac{N^{2}}{d^{2}}$ in the energies corresponds to the radial confinement of the particles. We assume the identical thickness $d$ for the both nanotubes, and that $d$ is sufficiently smaller than the radii of the nanotubes $d<<R_{\min }, R_{\max }$. Within this assumption below we use only the lowest energy state for the radial component of the eigenenergy $N=1$. Note the asymmetry between the electron and hole excitation spectra exist in the system due to different radii of the nanotubes, even for equal masses of the electrons and holes, $m_{e}=m_{h}$. The nonzero order parameter $\Delta_{\mathbf{p}, \mathbf{p}^{\prime}}$ shows that the system is in the superfluid phase. One has to keep in mind that it is assumed the electron-hole pairing occurs under the condition of the zero angular momentum of the pair. It results in canceling the linear momenta components for the electron and hole $\hbar k_{z}+\hbar k_{z}^{\prime}=0$, and also canceling their angular momenta: $L_{e}+L_{h}=0$. The latter condition leads immediately to $m+m^{\prime}=0$.

Let's diagonalize Eq.(11) using Bogoliubov unitary transformations $a_{\mathbf{p}}=u_{\mathbf{p}} \alpha_{-p}^{\dagger}+v_{\mathbf{p}} \beta_{-p}^{\dagger}$ and $b_{\mathbf{p}}=u_{\mathbf{p}} \beta_{\mathbf{p}}-$ $v_{\mathbf{p}} \alpha_{\mathbf{p}}$, with the amplitudes $v_{\mathbf{p}}$ and $u_{\mathbf{p}}$. Following Ref $\underline{\underline{3}}$, we assume that the order parameter is independent on the momentum: $\Delta_{\mathbf{p}, \mathbf{p}^{\prime}} \equiv \Delta$. Thus, the self-consistency condition for the order parameter has the form ${ }^{1}$

$$
\Delta=\frac{U}{S_{e f f}} \sum_{\mathbf{p}} u_{\mathbf{p}} v_{\mathbf{p}}\left(1-f\left(E_{+}\right)-f\left(E_{-}\right)\right)
$$

where $U$ is the effective attractive interaction between electrons and holes, and $S_{\text {eff }}=\pi L \sqrt{R_{\min } R_{\max }}$. Here we use the notation $E_{ \pm}=E_{\mathbf{p}} \pm \eta_{\mathbf{p}}, E_{\mathbf{p}}=\sqrt{\epsilon_{\mathbf{p}}^{2}+\Delta^{2}}$, $\epsilon_{\mathbf{p}}=\left(\xi_{\mathbf{p}}+\xi_{\mathbf{p}}^{\prime}-\mu_{e}-\mu_{h}\right) / 2, \eta_{\mathbf{p}}=\left(\xi_{\mathbf{p}}-\mu_{e}-\xi_{\mathbf{p}}^{\prime}+\mu_{h}\right) / 2$. In Eq.(4) Fermi-Dirac distribution function is given by $f(\epsilon)=\left[\exp \left(\epsilon /\left(k_{B} T\right)\right)+1\right]^{-1}$, where $k_{B}$ is Boltzmann constant, and $T$ is temperature. The amplitudes $u_{\mathbf{p}}, v_{\mathbf{p}}$ are given by

$$
\begin{aligned}
& u_{\mathbf{p}}^{2}=\frac{1}{2}\left(1+\frac{\epsilon_{\mathbf{p}}}{E_{\mathbf{p}}}\right), \\
& v_{\mathbf{p}}^{2}=\frac{1}{2}\left(1-\frac{\epsilon_{\mathbf{p}}}{E_{\mathbf{p}}}\right) .
\end{aligned}
$$

Note that a dielectric with a high dielectric constant between the nanotubes can significantly reduce the effective interaction $U$ that will dramatically reduce the BCS transition temperature $T_{c}$. The similar effect is obtained in the case of the increase of the separation distance $D=R_{\max }-R_{\min }$ between the nanotubes. For two axial nanotubes with a relatively small separation distance $D \ll R_{\min }, R_{\max }$ on can use the result for the interaction potential obtained for the electron-hole pairing in two parallel plane layer, given by Eq.(7) in Ref. $\stackrel{1}{\text {. As- }}$ suming the constant attraction of the paired particles one can use the simplified expression $U=\frac{e^{2}}{2 \varepsilon \varepsilon_{0} k_{F}} \exp \left(-D k_{F}\right)$, where $k_{F}$ is the Fermi wavevector, $\varepsilon$ is the characteristic dielectric constant of the dielectric between the coaxial nanotubes. The cut-off in the sum given by Eq. (44) is set by the characteristic plasma frequency $\stackrel{\underline{1}}{\underline{*}}$. The chemical potentials for electrons $\mu_{e}$ and holes $\mu_{h}$ are coupled self-consistently to the given surface densities of the carriers $\rho_{e, h}=N_{e, h} / S_{e, h} . \quad N_{e}$ and $N_{h}$ are the numbers of electrons and holes, respectively, while the surface of the outer nanotube is $S_{e}=2 \pi R_{\max } L$, the surface of the inner nanotube is $S_{h}=2 \pi R_{\min } L$, and $L$ is the length of each nanotube. Making the statistical averaging for the operators $\hat{N}_{h}=\sum_{\mathbf{p}} a_{\mathbf{p}}^{\dagger} a_{\mathbf{p}}$ and $\hat{N}_{e}=\sum_{\mathbf{p}} b_{\mathbf{p}}^{\dagger} b_{\mathbf{p}}$, one ob- 
tains

$$
\begin{aligned}
& \rho_{e}=\frac{2}{S_{e}} \sum_{\mathbf{p}}\left[f\left(E_{+}\right) u_{\mathbf{p}}^{2}+\left(1-f\left(E_{-}\right)\right) v_{\mathbf{p}}^{2}\right], \\
& \rho_{h}=\frac{2}{S_{h}} \sum_{\mathbf{p}}\left[f\left(E_{-}\right) u_{\mathbf{p}}^{2}+\left(1-f\left(E_{+}\right)\right) v_{\mathbf{p}}^{2}\right] .
\end{aligned}
$$

In our simulations we assume the electron surface density $\rho_{e}$ is fixed on the outer nanotube and the chemical potentials $\mu=\mu_{e}=\mu_{h}$ are equal. Then $\mu, \Delta$ and $\rho_{h}$ are determined by the simultaneous solution of Eqs. (4) and (6). Since the chemical potential and the order parameter mutually depend on each other, one needs to run through several iterations. Note, for metals the chemical potential is usually larger by many orders of magnitude than the order parameter. This significantly simplifies the calculations, because the increase of the order parameter below the critical temperature practically does not affect the chemical potential. So one can safely calculate the chemical potential by setting the order parameter to zero, i.e. in the normal state. For the system under consideration the order parameter value can reach up to $10 \%$ of the chemical potential, that requires the full selfconsistent solution of the problem. The calculations stop when the convergence condition is met. The chemical potential and the order parameter has to satisfy the condition, that for two consequent iterations the variations of their values are less than $0.1 \%$. It usually takes about several dozens of iterations to converge.

\section{CALCULATION OF THE CONDUCTIVITIES}

For the system of two coaxial nanotubes we introduce $\sigma_{e e}$ and $\sigma_{h h}$ as the longitudinal quasiparticle conductivities for electron and hole nanotubes, and $\sigma_{h e}$ and $\sigma_{e h}$ as the quasiparticle transconductivities. The quasiparticle conductivities can be obtained using Gorkov-Nambu Green's function formalism $3,5,23$ that gives the following expressions for the electron

$$
\sigma_{e e}=-\frac{e^{2}}{S_{e}} \sum_{\mathbf{p}} \frac{\pi|\mathbf{p}|^{2}}{m_{e}^{2}} \int_{-\infty}^{+\infty} \frac{\partial f(\epsilon)}{\partial \epsilon} A_{e e}^{2}(\mathbf{p}, \epsilon) d \epsilon
$$

and hole conductivities:

$$
\sigma_{h h}=-\frac{e^{2}}{S_{h}} \sum_{\mathbf{p}^{\prime}} \frac{\pi\left|\mathbf{p}^{\prime}\right|^{2}}{m_{h}^{2}} \int_{-\infty}^{+\infty} \frac{\partial f(\epsilon)}{\partial \epsilon} A_{h h}^{2}\left(\mathbf{p}^{\prime}, \epsilon\right) d \epsilon .
$$

Note, that the summation over the momenta $\mathbf{p}$ and $\mathbf{p}^{\prime}$ are not equivalent, because of the different quantization of the angular part for the inner and outer nanotubes. The transconductivities are obtained in a similar way:

$$
\sigma_{i j}=\frac{e^{2}}{S_{i}} \sum_{\mathbf{p}, \mathbf{p}^{\prime}} \frac{\pi \mathbf{p p}^{\prime}}{m_{i} m_{j}} \int_{-\infty}^{+\infty} \frac{\partial f(\epsilon)}{\partial \epsilon} A_{i j}^{2}(\mathbf{p}, \epsilon) d \epsilon
$$

where $(i, j)=(e, h)$ are the indices corresponding to the outer or inner nanotubes and the summation is done over the momenta with matching quantum numbers. Note that the momenta $\mathbf{p}$ and $\mathbf{p}^{\prime}$ contain the continuous linear $p_{z}, p_{z}^{\prime}$ and the discrete angular $p_{\phi}, p_{\phi}^{\prime}$ components in Eq. (9).

The matrix spectral function $\hat{A}(\mathbf{p}, \epsilon)$ is given by

$$
\hat{A}(\mathbf{p}, \omega)=-\frac{1}{\pi} \operatorname{Im} \hat{G}(\mathbf{p}, \epsilon+i \delta),
$$

where $\hat{G}$ is the Gorkov-Nambu matrix Green's function, and $\delta$ is an infinitesimal positive energy. In the absence of disorder we have $\hat{G}=\hat{G}^{0}$, and $\hat{G}^{0}$ determines the unperturbed spectral functions:

$$
\begin{array}{r}
A_{e e}^{0}(\mathbf{p}, \epsilon)=v_{\mathbf{p}}^{2} \delta\left(\epsilon-E_{+}\right)+u_{\mathbf{p}}^{2} \delta\left(\epsilon+E_{-}\right), \\
A_{h h}^{0}(\mathbf{p}, \epsilon)=u_{\mathbf{p}}^{2} \delta\left(\epsilon-E_{+}\right)+v_{\mathbf{p}}^{2} \delta\left(\epsilon+E_{-}\right), \\
A_{e h}^{0}(\mathbf{p}, \epsilon)=\left(\frac{\Delta}{2 E_{\mathbf{p}}}\right)\left(\delta\left(\epsilon-E_{+}\right)-\delta\left(\epsilon+E_{-}\right)\right) .
\end{array}
$$

In the presence of weak impurities the spectral functions become ${ }^{23}$ :

$$
\begin{array}{r}
A_{e e}(\mathbf{p}, \epsilon)=v_{\mathbf{p}}^{4} t_{h} \delta\left(\epsilon-E_{+}\right)+u_{\mathbf{p}}^{4} t_{e} \delta\left(\epsilon+E_{-}\right), \\
A_{h h}(\mathbf{p}, \epsilon)=u_{\mathbf{p}}^{4} t_{h} \delta\left(\epsilon-E_{+}\right)+v_{\mathbf{p}}^{4} t_{e} \delta\left(\epsilon+E_{-}\right), \\
A_{e h}(\mathbf{p}, \epsilon)=\left(\frac{\Delta}{2 E_{\mathbf{p}}}\right)\left(t_{h} \delta\left(\epsilon-E_{+}\right)+t_{e} \delta\left(\epsilon+E_{-}\right)\right) .
\end{array}
$$

In the presence of impurities the conductivity for the outer nanotube $\sigma_{e e}$ can be calculated as

$$
\begin{array}{r}
\sigma_{e e}=-\frac{e^{2} \pi}{S_{e}} \sum_{p_{z}, p_{\phi}} \frac{p_{z}^{2}+p_{\phi}^{2}}{m_{e}^{2} k_{B} T} \times \\
{\left[v_{\mathbf{p}}^{4} \frac{t_{h}}{\cosh ^{2}\left(\frac{E_{+}}{2 k_{B} T}\right)}+u_{\mathbf{p}}^{4} \frac{t_{e}}{\cosh ^{2}\left(\frac{E_{-}}{2 k_{B} T}\right)}\right] .}
\end{array}
$$

To evaluate this expression, the $p_{z}$ was quantized using $p_{z}=l \hbar / L$ where $l=0, \pm 1, \pm 2, \ldots$ In the simulations the length of the nanotube $L$ is set to be relatively big to simulate an infinite system, particularly $L=1000 \mathrm{~nm}$. Similarly one can represent the conductivity for the inner nanotube $\sigma_{h h}$ :

$$
\begin{array}{r}
\sigma_{h h}=-\frac{e^{2} \pi}{S_{h}} \sum_{p_{z}^{\prime}, p_{\phi}^{\prime}} \frac{p_{z}^{\prime 2}+p_{\phi}^{\prime 2}}{m_{h}^{2} k_{B} T} \times \\
{\left[u_{\mathbf{p}}^{4} \frac{t_{h}}{\cosh ^{2}\left(\frac{E_{+}}{2 k_{B} T}\right)}+v_{\mathbf{p}}^{4} \frac{t_{e}}{\cosh ^{2}\left(\frac{E_{-}}{2 k_{B} T}\right)}\right] .}
\end{array}
$$

The interlayer transconductivity $\sigma_{e h}$ can be similarly ex- 
pressed as

$$
\begin{gathered}
\sigma_{e h}=\frac{e^{2} \pi}{S_{e}} \sum_{p_{z}, p_{z}^{\prime}, p_{\phi}, p_{\phi}^{\prime}} \frac{p_{z} p_{z}^{\prime}+p_{\phi} p_{\phi}^{\prime}}{m_{e} m_{h} k_{B} T} \times \\
{\left[\frac{t_{h}}{\cosh ^{2}\left(\frac{E_{+}}{2 k_{B} T}\right)}+\frac{t_{e}}{\cosh ^{2}\left(\frac{E_{-}}{2 k_{B} T}\right)}\right] u_{\mathbf{p}}^{2} v_{\mathbf{p}}^{2}}
\end{gathered}
$$

The corresponding transconductivity $\sigma_{h e}=\sigma_{e h} \frac{R_{\max }}{R_{\min }}$ because in the derivation of the Kubo's formula 23 the induced current should be averaged over the area where it is flowing. Therefore, since the transconductivity $\sigma_{e h}$ describes the current of electrons on the outer nanotube induced by the holes current on the inner nanotube, the normalization area should be $S_{e}$, that is reflected in Eq. (91). Note the asymmetry in the transconductivities $\sigma_{e h} \neq \sigma_{h e}$ exists only for the spatially separated electrons and holes in two colaxial nanotubes due to the difference between their radii, while the relation $\sigma_{e h}=\sigma_{h e}$ holds for two parallel plane layers ${ }^{3}$. Follow Ref. 3 the scattering lifetimes of quasiparticles can be evaluated as:

$$
\begin{aligned}
& t_{h}^{-1}=t_{h n}^{-1} u_{\mathbf{p}}^{4}\left|\frac{\partial E_{+}}{\partial \xi_{\mathbf{p}}^{\prime}}\right|^{-1}+t_{e n}^{-1} v_{\mathbf{p}}^{4}\left|\frac{\partial E_{+}}{\partial \xi_{\mathbf{p}}}\right|^{-1} \\
& t_{e}^{-1}=t_{h n}^{-1} v_{\mathbf{p}}^{4}\left|\frac{\partial E_{-}}{\partial \xi_{\mathbf{p}}^{\prime}}\right|^{-1}+t_{e n}^{-1} u_{\mathbf{p}}^{4}\left|\frac{\partial E_{-}}{\partial \xi_{\mathbf{p}}}\right|^{-1} .
\end{aligned}
$$

In Eq. (16) $t_{e n}$ and $t_{h n}$ are the electron and hole scattering times in the normal state. The electron and hole scattering times $t_{e n}$ and $t_{h n}$ are related to the hole $V_{\mathbf{p p}^{\prime}}^{h}$ and electron $V_{\mathbf{p p}^{\prime}}^{e}$ impurities scattering potentials as follows:

$$
\begin{aligned}
t_{h n}^{-1} & =4 \pi \sum_{\mathbf{p}^{\prime}}<\left|V_{\mathbf{p p}^{\prime}}^{h}\right|^{2}>\delta\left(\xi_{\mathbf{p}}^{\prime}-\xi_{\mathbf{p}^{\prime}}^{\prime}\right), \\
t_{e n}^{-1} & =4 \pi \sum_{\mathbf{p}^{\prime}}<\left|V_{\mathbf{p} \mathbf{p}^{\prime}}^{e}\right|^{2}>\delta\left(\xi_{\mathbf{p}}-\xi_{\mathbf{p}^{\prime}}\right),
\end{aligned}
$$

where $\langle.$.$\rangle denotes the statistical average over the in-$ purities. It is assumed that the impurieties on the inner and outer nanotubes are uncorrelated: $\left\langle V^{e} V^{h}\right\rangle=0$. Using the chain rule one obtains: $\left|\frac{\partial E_{+}}{\partial \xi_{\mathrm{p}}}\right|=\left|\frac{\epsilon_{\mathrm{p}}}{E_{\mathrm{p}}} y+y_{1}\right|$, $\left|\frac{\partial E_{-}}{\partial \xi_{\mathbf{p}}}\right|=\left|\frac{\epsilon_{\mathrm{p}}}{E_{\mathrm{p}}} y-y_{1}\right|,\left|\frac{\partial E_{+}}{\partial \xi_{\mathrm{p}}^{\prime}}\right|=\left|\frac{\epsilon_{\mathrm{p}}}{E_{\mathrm{p}}} y_{2}+y_{3}\right|$ and $\left|\frac{\partial E_{-}}{\partial \xi_{\mathrm{p}}^{\prime}}\right|=$ $\left|\frac{\epsilon_{\mathbf{p}}}{E_{\mathbf{p}}} y_{2}-y_{3}\right|$. Here we used a notation $y=\frac{1}{4}(2+x+x w)$, $y_{1}=\frac{1}{4}(x+x w-2), y_{2}=\frac{1}{4}\left(2+x^{-1}+(x w)^{-1}\right), y_{3}=$ $\frac{1}{4}\left(x^{-1}+(x w)^{-1}-2\right)$, where $x=\frac{m_{e}}{m_{h}}$ and $w=\frac{R_{\max }^{2}}{R_{\min }^{2}}$. Note that for $R_{\min }=R_{\max }$ Eq. (16) reduces to the expression given by Eq. 16 in Ref 3 . Since we are interested to study the effects of the asymmetry between electrons and holes due to different radii of the nanotubes, in our simulations we assume the electrons and holes have equal masses: $m_{e}=m_{h}=m$. We also assume that the scattering times of electrons and holes in the normal state are equal: $t_{e n}=t_{h n}=t_{n}$. Since the electron and hole scattering times enter the conductivities as linear weighting

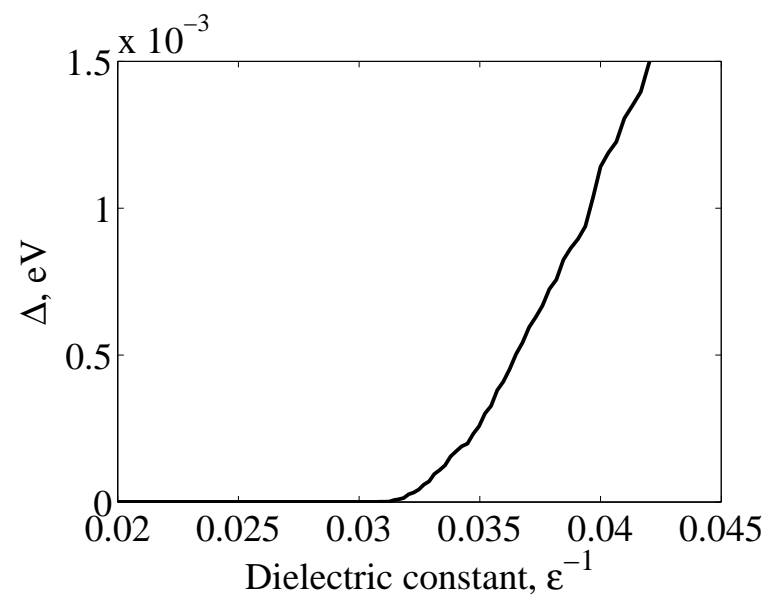

FIG. 2: The order parameter $\Delta$ as a function of the inverse dielectric constant $\varepsilon^{-1}$. The radii of the nanotubes are $R_{\min }=6 \mathrm{~nm}$ and $R_{\max }=8 \mathrm{~nm}$. The surface carrier concentration is $\rho=10^{12} \mathrm{~cm}^{-2}$ and the temperature is $T=0 \mathrm{~K}$.

coefficients, we do not expect any qualitative change of our results in the case of unequal scattering times. Similar conclusion was expressed also in Ref. 3, where different electron and hole masses and lifetimes were used. Let us emphasize that the transconductivities $\sigma_{e h}$ and $\sigma_{h e}$ are negative $e^{-}$, because for a system of spatially separated charges the Coulomb drag induces the currents flowing in the opposite directions.

In general, the currents are carried by the BCS superfluid and quasiparticles ${ }^{3}$. We characterize the quasiparticle contributions in the linear-response regime as follows:

$$
\left(\begin{array}{c}
j_{h} \\
j_{e}
\end{array}\right)=\left(\begin{array}{cc}
\sigma_{h h} & \sigma_{h e} \\
\sigma_{e h} & \sigma_{e e}
\end{array}\right)\left(\begin{array}{c}
E_{h} \\
E_{e}
\end{array}\right)
$$

where $j_{e(h)}$ and $E_{e(h)}$ are the current flows of quasiparticles and electric field in electron (hole) nanotubes, correspondingly.

Let us mention that the electric fields in electron and hole nanotubes are identical ${ }^{3}: E \equiv E_{e}=E_{h}$. Therefore from Eq. (18) we obtain

$$
E=\frac{j_{e}+j_{h}}{\sigma_{e e}+\sigma_{h h}+\sigma_{h e}+\sigma_{e h}}
$$

Let us consider the drag setup, where a fixed current $j_{0}$ flows through the drive nanotube and the voltage drop across the second nanotube, namely the drag-nanotube, is measured, $j_{h 0}=j_{0}$ and $j_{e 0}=0$. Using Eq. (19) for $j_{e}+j_{h}=j_{0}$, the voltage in the drag nanotube $V_{d r a g}$ can be written as

$$
V_{d r a g}=L E=\frac{L j_{0}}{\sigma_{e e}+\sigma_{h h}+\sigma_{h e}+\sigma_{e h}} .
$$




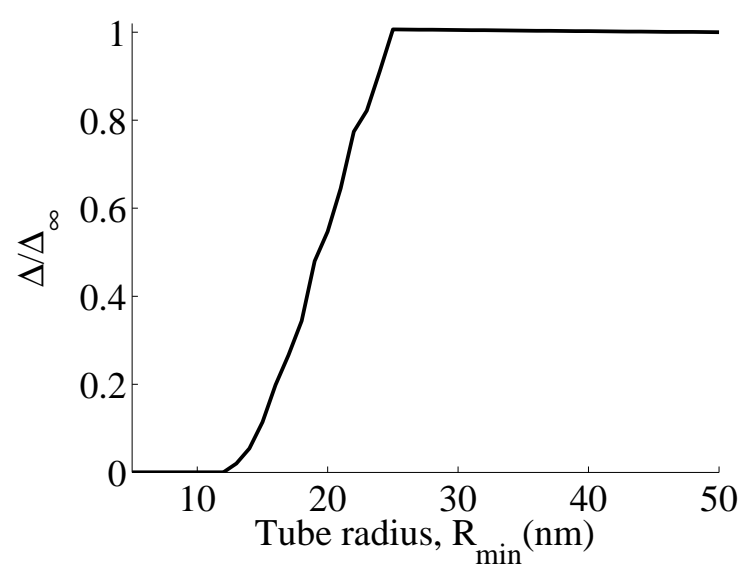

FIG. 3: The normalized order parameter $\Delta$ as function of the radius of the inner nanotube $R_{\text {min }}$. The radii difference $D=$ $R_{\max }-R_{\min }$ is kept constant $D=2 \mathrm{~nm}$. The surface carrier concentration is $\rho=10^{12} \mathrm{~cm}^{-2}$ and the temperature is $T=0 \mathrm{~K}$. The order parameter is normalized to its value $\Delta_{\infty}$ for the flat layers geometry, that corresponds to $R_{\min }, R_{\max } \rightarrow \infty$.

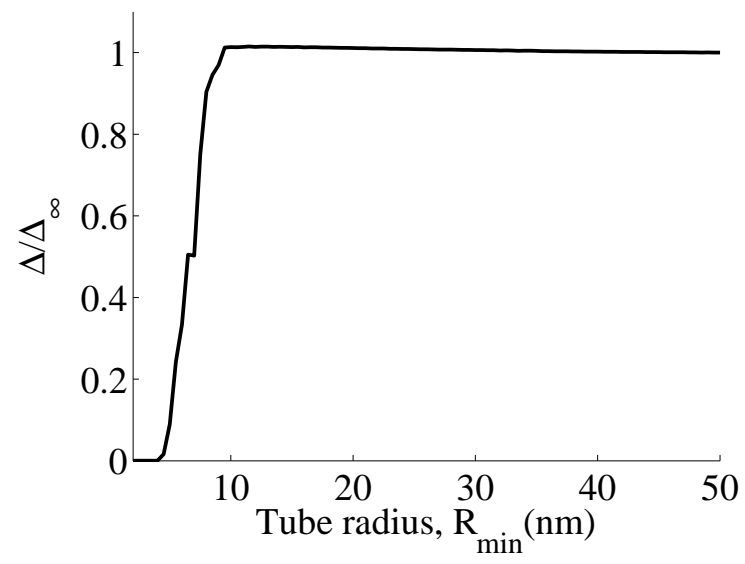

FIG. 4: The normalized order parameter $\Delta$ as function of the radius of the inner nanotube $R_{\min }$. Note the electron-hole interaction is $50 \%$ stronger than in Fig. 3 The radii difference $D=R_{\max }-R_{\min }$ is kept constant: $D=2 \mathrm{~nm}$. The surface carrier concentration is $\rho=10^{12} \mathrm{~cm}^{-2}$ and the temperature $T=0 \mathrm{~K}$. The order parameter is normalized to its value $\Delta_{\infty}$ for the flat layers geometry, that corresponds to $R_{\min }, R_{\max } \rightarrow \infty$.

\section{DISCUSSION AND CONCLUSIONS}

For all presented calculations the surface electron concentration is set to $10^{12} \mathrm{~cm}^{-2}$. We have also performed calculations using much higher density, $10^{14} \mathrm{~cm}^{-2}$. We have found that the increase of the density does not lead to qualitative changes of the presented in this work results. The hole concentration is obtained self-consistently and under the condition of equal chemical potentials it is very close to the concentration of electrons.

First, we study the role of electron-hole attraction in

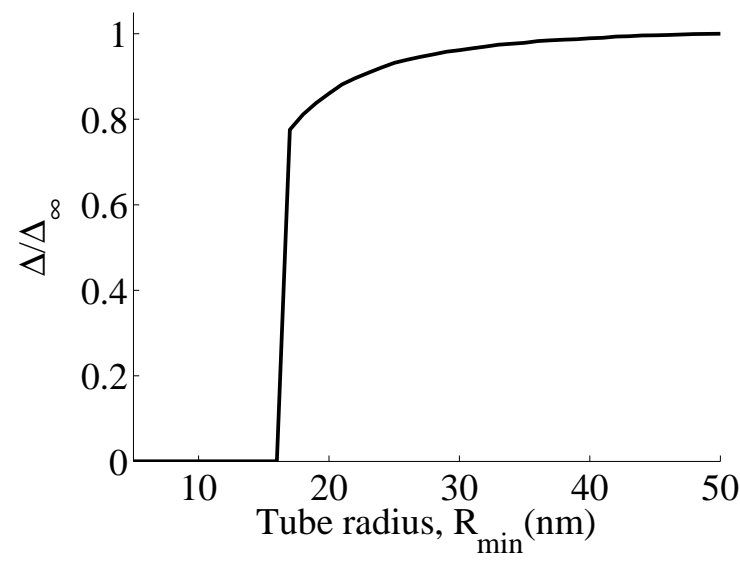

FIG. 5: The normalized order parameter $\Delta$ as a function of the radius of the inner nanotube $R_{\min }$. The electron-hole interaction is the same as in Fig. 4 The radii difference $D=R_{\max }-R_{\min }$ is kept constant $D=2 \mathrm{~nm}$. The surface carrier concentration is $\rho=10^{12} \mathrm{~cm}^{-2}$ and the temperature is $T=0.5 T_{c}$, where $T_{c}$ is the critical temperature for the flat layers geometry. The order parameter is normalized to its value $\Delta_{\infty}$ for the flat layers geometry, that corresponds to $R_{\min }, R_{\max } \rightarrow \infty$.

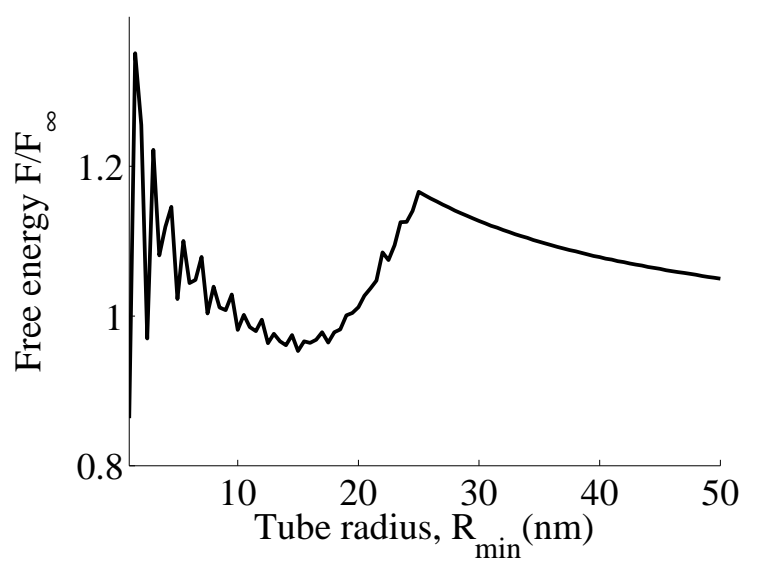

FIG. 6: The normalized free energy density as function of the radius of the inner nanotube $R_{\min }$. The radii difference $D=$ $R_{\max }-R_{\min }$ is kept constant $D=2 \mathrm{~nm}$. The surface carrier concentration is $\rho=10^{12} \mathrm{~cm}^{-2}$ and the temperature is $T=0 \mathrm{~K}$. The free energy is normalized to its value $F_{\infty}$ for the flat layers geometry, that corresponds to $R_{\min }, R_{\max } \rightarrow \infty$.

the system on the formation of the superfluid phase. It is known that in the case of the asymmetry caused by the difference between the masses of the electrons and holes there is the critical strength of the electron-hole attraction, under which there is no BCS transition 24 . In a system of two coaxial nanotubes the strength of interaction can be controlled, for example, by changing the dielectric constant of the dielectric between the nanotubes. To illustrate this we calculate the dependence of the order parameter on the inverse dielectric constant 


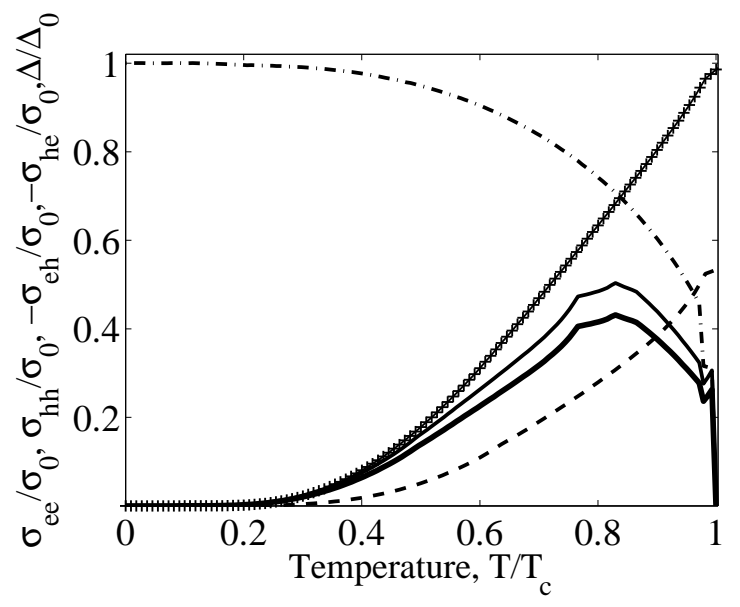

FIG. 7: The normalized quasiparticles conductivities $\sigma_{e e}$ (line with the plus markers), $\sigma_{h h}$ (dashed line), $\sigma_{e h}$ (thin solid line), $\sigma_{h e}$ (thick solid line) and the order parameter $\Delta$ (dash-dotted line) as functions of the normalized temperature $T / T_{c}$. The radii of the nanotubes are $R_{\min }=5 \mathrm{~nm}$ and $R_{\max }=7 \mathrm{~nm}$, the surface carrier concentration is $\rho=10^{12} \mathrm{~cm}^{-2}$. Note the conductivities are normalized to the electron conductivity of the outer nanotube $\sigma_{0}$ at the normal state, and the order parameter is normalized to its value $\Delta_{0}$ at $T=0 \mathrm{~K}$.

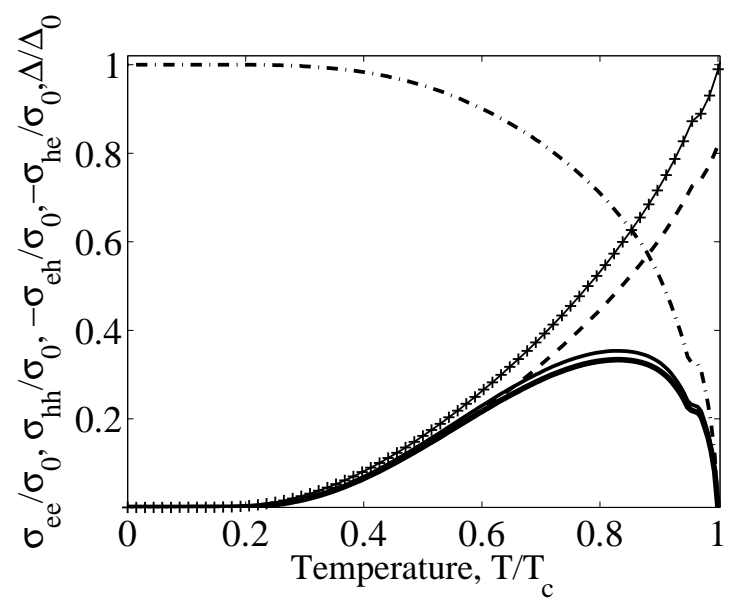

FIG. 8: The normalized quasiparticles conductivities $\sigma_{e e}$ (line with the plus markers), $\sigma_{h h}$ (dashed line), $\sigma_{e h}$ (thin solid line), $\sigma_{h e}$ (thick solid line) and the order parameter $\Delta$ (dash-dotted line) as functions of the normalized temperature $T / T_{c}$. The radii of the nanotubes are $R_{\min }=50 \mathrm{~nm}$ and $R_{\max }=52 \mathrm{~nm}$, the surface carrier concentration is $\rho=10^{12} \mathrm{~cm}^{-2}$. Note the conductivities are normalized to the electron conductivity of the outer nanotube $\sigma_{0}$ at the normal state, and the order parameter is normalized to the order parameter $\Delta_{0}$ at $T=0 \mathrm{~K} . \sigma_{h e}$ and $\sigma_{e h}$ are so close that they are indistinguishable on this figure.

presented in Fig. 2. From Fig. 2 2 one can see that there is a critical value for the interaction strength. For a weaker electron-hole interaction strength (or higher value of the dielectric constant) there is no BCS state. In our calculations the radii of the nanotubes are set to $R_{\min }=6 \mathrm{~nm}$ and $R_{\max }=8 \mathrm{~nm}$ and the temperature $T=0 \mathrm{~K}$. Note that for different radii of the nanotubes the asymmetry between electrons and holes will be different, that will correspond to different critical values of the interaction strength. In general, for a larger asymmetry one would expect a higher critical value of the interaction strength. For the symmetric case of two parallel plane layers the change in attraction strength between the carriers results in the change of the gap, which follows the standard BCS theory.

Second, we investigate how relatively large electronhole asymmetry can destroy the superfluid phase in the system. The results of the calculations for the dependence of the normalized order parameter on the radius of the inner nanotube for the different strength of the electron-hole interaction and for different temperature are presented in Figs. 3- 5 . According to the results of our calculations, the BCS state still can be formed in the case of moderate asymmetry between the electrons and holes excitation spectra. By decreasing the radii $R_{\text {min }}$ and $R_{\max }$ of the nanotubes while keeping the constant separation distance $D=R_{\max }-R_{\min }$ between the nanotubes one can monotonously increase the asymmetry in the system. In the simulations shown in Figs. 3, 4 and 5 the separation distance is set to $D=2 \mathrm{~nm}$ and the dielectric constant between the nanotubes is set to $\varepsilon=27$. As it seen from Fig. 3 for relatively large radii $\left(R_{\min }, R_{\max }>25 \mathrm{~nm}\right)$ the order parameter in the system converges to its value for the plane layers system. At zero temperature $T=0 \mathrm{~K}$, as the radii decrease, the order parameter has a kink at $\approx 25 \mathrm{~nm}$ and then almost linearly drops to zero for the inner radius of the nanotube $12 \mathrm{~nm}<R_{\min }<25 \mathrm{~nm}$. One can suggest that for a fixed attraction strength between electrons and holes there is a critical asymmetry, and that for a higher degree of the asymmetry there is no BCS transition. The nature of the kink in Fig. 3 has to be studied in more details. The oscillations in the dependence of the order parameter and critical temperature on the size of superconducting nanowires ${ }^{25,26}$, superconducting films $\frac{26}{}$, superconducting metallic grains 27 were obtained in the framework of BCS and Bogoliubov-de Gennes theories caused by quantum size effect. The experimental demonstration of the quantum size effects in the dependence of the critical temperature on the size of the superconducting film was observed in Ref. 28. The similar kink to the one obtained in our Paper for the ground state energy respect to the coupling constant was treated as a firstorder quantum phase transition in the orthogonal-dimer spin chain.$^{29}$. The possibility of the first order quantum phase transition resulting in the point of non-analyticity in the ground energy as a function of the nanotube radius will be studied in the future.

To further investigate the effect of the electron-hole asymmetry in Fig. 6 we show the normalized free energy density in the system as a function of the inner radius. At zero temperature the free energy is equal to the ground state energy of the system. For the calculations the difference $R_{\max }-R_{\min }=2 \mathrm{~nm}$ is kept constant. 
The dielectric constant and temperature are the same as for Fig. 3. The free energy density has also a kink at the radius $R_{\min } \approx 25 \mathrm{~nm}$, which is the same radius, at which the kink in the order parameter is observed in Fig. 3. This should not be a surprise, since the ground state of a BCS superconductor contains a term proportional to $\Delta^{2 \underline{5}}$. The oscillations in the dependence of the ground state energy on the nanotube radius $R_{\text {min }}$ for $R_{\text {min }}<25 \mathrm{~nm}$ seems to be caused by the quantum size effects.

For a stronger attraction between the electrons and holes, which is modeled by a dielectric with 1.5 times smaller dielectric constant $\varepsilon=18$, the system can sustain a higher degree of the asymmetry that is shown in Fig. 4. At $T=0 \mathrm{~K}$ the order parameter increases essentially with the decrease of the radii of the nanotubes. This effect is attributed to the quantum confinement and it was predicted for homogeneous $\frac{11}{}$ and inhomogeneous ${ }^{30}$ superconducting nanowires. For the radii less than the critical radii $R_{\text {min }} \approx 10 \mathrm{~nm}$ the order parameter again shows an almost linear drop to zero.

The similar drop can be observed at the finite temperature $T=0.5 T_{c}$ presented in Fig. 5 , where $T_{c}$ is the critical temperature for the plane layers system. In Fig. 5 the critical radius $R_{\text {min }} \approx 17 \mathrm{~nm}$ is considerably bigger than the critical radius for $T=0 \mathrm{~K}$. One can also notice that the enhancement of the order parameter due to the quantum confinement does not take place at this sufficiently high temperature.

Finally, we study the temperature dependence of the transconductivity coefficients in the system. We have found there are non-zero drag conductivities $\sigma_{e h} \neq \sigma_{h e}$ between the two coaxial nanotubes at temperatures below the critical temperature of the BCS phase transition $T_{c}$. Since the drag conductivity is a signature of the BCS state, the measurements of the drag conductivity can be used to demonstrate the existence of the BCS state resulting in the electron-hole superfluidity in the system and superconductivity in each nanotube. The measurement of the temperature corresponding to vanishing of the drag conductivity can be treated as the measurement of $T_{c}$. Note in the limiting case of low carrier concentrations the exciton pairs will be formed Ref.15, that will result in a finite drag even at high temperatures due to the Coulomb attraction and formation of bounded states for electron-hole pairs. The results of calculation of the order parameter and the normalized conductivity coefficients as functions of the temperature are presented in Figs. 7 and 8 The conductivity coefficients are normalized to the conductivity of the outer nanotube in the normal state $\sigma_{e n}$, and, therefore, they do not depend on the scattering time of the carriers, which is assumed equal for electrons and holes.

To understand the dependence of the conductivities and the order parameter on temperature we considered two scenarios and perform calculations for two systems when the radii of the nanotubes are different by the order of magnitude, however the separation distance be- tween the inner and outer cylinders was kept constant: $D=2 \mathrm{~nm}$. Particularly, we considered the system when the inner nanotube has $R_{\min }=5 \mathrm{~nm}$ and the outer one has $R_{\text {min }}=7 \mathrm{~nm}$ and a larger system with $R_{\text {min }}=50 \mathrm{~nm}$ and $R_{\min }=52 \mathrm{~nm}$. The results of calculations are presented in Figs. 7 and 8, correspondingly. Note that we plot the conductivities in the units of the conductivity of the outer nanotube $\sigma_{e n}$ in the normal state. The comparison of the results of the calculations demonstrates that as the temperature $T$ approaches the critical temperature of the phase transition $T_{c}$, the transconductivities $\sigma_{e h}$ and $\sigma_{h e}$ vanish, because the quasiparticle drag conductivity appears due to the existence of the electronhole pairing caused by the Coulomb attraction, and when the order parameter $\Delta$ vanishes, the conductivities $\sigma_{e h}$ and $\sigma_{h e}$ also vanish. At low temperatures the conductivities are exponentially suppressed. The similar behavior was obtained for an infinite two plane layer electron-hole system in Ref. 3 . We also would like to emphasize that $\sigma_{e e} \neq \sigma_{h h}$ due to the difference between the radii of the nanotubes, and the quasiparticle transconductivity $\sigma_{e h}$ takes values of the same order of magnitude as $\sigma_{e e}$ and $\sigma_{h h}$. The difference between the transconductivities $\sigma_{e h}$ and $\sigma_{h e}$ is also the specific property of the spatially separated electrons and holes in two coaxial nanotubes, while for two plane electron and hole layers one has $\sigma_{e h}=\sigma_{h e} e^{3}$. Note that in Figs. 7 and 8 the normalized gap function has little smooth kinks at some temperatures. This corresponds to the mutual effects between the order parameter and the chemical potential in the system. Unlike in superconducting metals, in our calculations the ratio $\Delta / \mu$ is of the order of 0.1 , so the changes of the gap with the temperature affects the chemical potential and through it the conductivity.

To summarize, a system of two coaxial nanotubes with spatially separated electrons and holes on each nanotube was studied theoretically. It was shown that at the temperatures lower than the critical temperature the system undergoes BCS-like transition. The transition results in correlations between the carriers on each nanotube and as a result, nonzero Coulomb drag coefficient. The measurement of the drag coefficient can be used to monitor the BCS transition in the system. We analyzed how the asymmetry between electrons and holes controlled through the radii of the nanotubes, affects the formation of the BCS state in the system. We found that there is a kink in the dependence of the order parameter and the ground state energy on the asymmetry with the monotonous change of the asymmetry in the system. For relatively stronger attraction between the electrons and holes and sufficiently low temperatures the system can exhibit the enhancement of the order parameter due to the quantum confinement effect. We also anticipate for a system of two coaxial nanotubes with equal carriers concentrations the contribution to the conductivity due to the unpaired component on the outer nanotube. The considered system has an intrinsic asymmetry due to different radii of the nanotubes. One can utilize this 
asymmetry in nanoscale devices. Unlike the ratio of the transconductivities $\sigma_{e h} / \sigma_{h e}$, for relatively small radii of the nanotubes the ratio $\sigma_{e e} / \sigma_{h h}$ is not equal to the ratio of radii, and therefore, the ratio of the amount of the carriers on the nanotubes. Therefore, a situation is possible where the inner drive nanotube can induce a higher current in the outer drag nanotube. In other words, the drag current will be higher than the drive one. The potential drop on the outer nanotube will be lower than on the inner one. Therefore, the system functions as a step down transformer at zero frequency. In the case of using the outer nanotube as the drive, the system works as a step up transformer, where the drag current will be lower than the drive current. In the case of ideal drag the transformation coefficient is given by the ratio of the radii of the nanotubes. One can possibly manufacture such systems using multiwall carbon nanotubes.

\section{Acknowledgments}

The authors are grateful to G. Vignale for valuable discussions. O.L.B. acknowledges the support of KITP, Santa Barbara under National Science Foundation Grant No. PHY-1125915, and their kind hospitality at the early stages of this project.
${ }^{1}$ Lozovik Yu E and Yudson V I 1976 Sov. Phys.-JETP 44 389.

2 Pogrebinskii M B 1977 Fiz. Tekh. Poluprovodn. 11637 Pogrebinskii M B 1977 Sov. Phys.Semicond. 11372 (Engl. transl.)

3 Vignale G and MacDonald A H 1996 Phys. Rev. Lett. 76 2786.

4 Balatsky A V, Joglekar Y N and Littlewood P B 2004 Phys. Rev. Lett. 93266801

${ }^{5}$ Schrieffer J R 1964 Theory of Superconductivity (New York: Benjamin)

${ }^{6}$ Nandi D, Finck A D K, Eisenstein J P, Pfeiffer L N and West K W 2012 Nature 488481

7 Spuntarelli A, Pieri P and Strinati G C 2010 Phys. Rep. 488111

8 Joglekar Y N, Balatsky A V and Lilly M P 2005 Phys. Rev. B 72205313

9 Berman O L, Kezerashvili R Ya and Lozovik Yu E 2010 Phys. Lett. A $\mathbf{3 7 4} 3681$

10 Berman O L, Kezerashvili R Ya and Lozovik Yu E 2010 Phys. Rev. B 82125307

11 Shanenko A A, Croitoru M D and Peeters F M 2007 Phys. Rev. B 75014519

12 Croitoru M D, Shanenko A A, Kaun C C and Peeters F M 2009 Phys. Rev. B 80024513

13 Bedaque P F, Caldas H and Rupak G 2003 Phys. Rev. Lett. 91247002 Caldas H 2004 Phys. Rev. A 69063602

142000 An Introduction to the Theory of Superfluidity (Boulder, Colorado: Westview Press) pp 14952

15 Deinega A, Voronova N and Lozovik Yu 2012 J. Phys.:
Condens. Matter 24255301

16 Finkelstein G, Shtrikman H and Bar-Joseph I 1995 Phys. Rev. Lett. 74976

17 Esser A, Runge E, Zimmermann R and Langbein W 2000 Phys. Rev. B 628232

18 Bar-Joseph I 2005 Semicond. Sci. Technol. 20 R29

19 Schuck P, Sogo T and Roepke G 2009 Int. J. Mod. Phys. A 242027

20 Fulde P and Ferrell R A 1964 Phys. Rev. A 135 A550

21 Larkin A I and Ovchinnikov Yu N 1964 Zh. Eksp. Teor. Fiz. 471136

Larkin A I and Ovchinnikov Yu N 1965 Sov. Phys.JETP 20762 (Engl. transl.)

22 Bigazzi F, Cotrone A L, Musso D, Pinzani Fokeeva N and Seminara D 2012 J. High Energy Phys. 120278

23 Mahan G D 1990 Many-Particle Physics (New York: Plenum) chapters 7 and 9

24 Conti S, Vignale G and MacDonald A H 1998 Phys. Rev. $B 57$ R6846

25 Shanenko A A, Croitoru M D, Zgirski M, Peeters F M and Arutyunov K 2006 Phys. Rev. B 74052502

26 Croitoru M D, Shanenko A A and Peeters F M 2007 Phys. Rev. B $\mathbf{7 6} 024511$

27 García-García A M, Urbina J D, Yuzbashyan E A, Richter K and Altshuler B L 2008 Phys. Rev. Lett. 100187001

28 Guo Y et al 2004 Science 3061915

${ }^{29}$ Koga A, Okunishi K and Kawakami N 2000 Phys. Rev. B 625558

30 Grigorenko I and Zhu J-X 2008 J. Phys.: Condens. Matter 20195204 